\title{
Modalidades y destinos turísticos del cantón Puerto Quito, provincia de Pichincha
}

Fecha de recepción : 20 de agosto de 2019 • Fecha de aceptación: 5 de septiembre de 2019 • Fecha de publicación: 10 de septiembre de 2019

Mg. Aníbal Gonzalo Fuentes Moreno Universidad Central del Ecuador afuentes@uce.edu.ec

Mg. Karol Andrea Rivas Ramírez Universidad Internacional del Ecuador karivas@uide.edu.ec

Evelyn Mikaela Arcentales Aulestia emarcentales@uce.edu.ec

Jessenia Marisol Salazar Aguilar jmsalazara1@uce.edu.ec

\section{Resumen}

El presente artículo tiene como objetivo identificar las modalidades y destinos turísticos del Cantón Puerto Quito, para lo cual se sistematizó la información disponible -en repositorios digitales, bibliografía especializada y artículos indexados que analizan sus definiciones y experiencias en el contexto mundial - que podría ser aplicada en el territorio de estudio. La investigación se sustentó en procedimientos de observación geográfica de campo, jerarquización de atractivos turísticos, identificación de ecosistemas, determinación de expresiones culturales, registro de empresas de servicios turísticos, así como en una encuesta dirigida a turistas. Se utilizó estadística descriptiva y se analizaron los datos obtenidos a través de barras y pasteles con el apoyo del software Excel. Los resultados obtenidos serán la base de planificación turística para el sector público, el sector privado, las comunidades locales y la academia que tengan en prospectiva generar proyectos en el territorio.

Palabras clave: atractivos, destinos, modalidades, tendencias, turismo. 


\begin{abstract}
The purpose of this paper is to identify the modalities and tourist destinations of the Puerto Quito Canton, for which the available information - in digital repositories, specialized bibliography and indexed articles that analyze their definitions and experiences in the global context - that could be applied in the field of study was systematized. The research was based on procedures of geographical field observation, ranking of tourist attractions, identification of ecosystems, determination of cultural expressions, registration of tourism services companies, as well as a survey aimed at tourists. Descriptive statistics were used and the data obtained through bars and pie charts were analyzed with the support of Excel software. The results obtained will be the basis of tourism planning for the public, private, local communities and the academy that has prospective projects in the territory.
\end{abstract}

Keywords: attractions, destinations, modalities, trends, tourism. 


\section{Introducción}

El turismo ha generado significativos indicadores de progreso en el ámbito socioeconómico, político, cultural, empresarial y ambiental, siendo una de las actividades que con mayor éxito dinamizan territorios a través de la sostenibilidad. El sector del turismo ha experimentado grandes cambios en los últimos veinte años, diversificándose la oferta de destinos y estableciéndose de manera más clara la planificación de modalidades turísticas o productos turísticos integrados para satisfacer a través de la operación turística a nuevas e imprevisibles tendencias turísticas.

Ecuador geográficamente se encuentra en un lugar estratégico que le ha permitido el desarrollo de una exuberante diversidad natural y cultural, siendo reconocido mundialmente como el país de los cuatro mundos (Ministerio de Turismo, 2004). Según la plataforma GEOVIT instaurada por el Ministerio de Turismo del Ecuador, hasta diciembre de 2018 se recibieron 1300000 turistas internacionales, sin tomar en cuenta al menos 810000 migrantes venezolanos que ingresaron con visa de turista buscando refugio y trabajo como consecuencia de la crisis humanitaria en su país de origen (GEOVIT, 2019). El ingreso por turismo genera el $2 \%$ del Producto Interno Bruto (PIB) de la economía del país ubicándose en el séptimo puesto entre las actividades económicas que aportan al desarrollo del país.

Tras identificarse el turismo como eje dinamizador de la economía del país, el Ministerio de Turismo como entidad rectora estableció en el año 2007 un Plan Estratégico de Desarrollo de Turismo en el Ecuador al año 2020 (PLANDETUR 2020) que proponía ocho objetivos generales para posicionar al país como un destino turístico sostenible líder que contribuya al logro de los Objetivos de Desarrollo del Milenio con elevada responsabilidad social corporativa y efectiva gestión socio-cultural y ambiental a través de un sistema moderno de gobernanza del sector turístico, con un esquema jurídico claro, un sistema de información eficiente y un marco institucional fortalecido que facilite el ejercicio de las actividades turísticas, que impulse el proceso de descentralización en implementación de infraestructura turística de apoyo además de la formación de talento humano especializado dirigidos a fortalecer tres líneas de productos así: Ecoturismo y Turismo de Naturaleza, Turismo Cultural y finalmente el Turismo de Deportes y Aventura (Ministerio de Turismo, 2007).

El Ministerio de Turismo de Ecuador determina, a través del Plan Nacional de Desarrollo Turístico Sostenible 2020, diez Destinos Turísticos Regionales (DTR) y dos Distritos Metropolitanos. Uno de los DTR es el de la Amazonía, de importancia económica estratégica por la construcción de la Ruta Manta-Coca-Manaos, Parque Nacional Yasuní y la Troncal Amazónica (MINTUREC, Actualización PLANDETUR 2020, 2015), consolidándose la región en los objetivos de desarrollo en los Planes Nacionales del Buen Vivir 2013 -2017 y 2017-2021, dando prioridad a las modalidades turísticas de ecoturismo y rural, con énfasis en el aprovechamiento turístico de 55 áreas protegidas y 23 grupos étnicos, en especial para las regiones Amazonía y Andina.

Para ello, a partir del 2007 se pone en marcha el Plan Estratégico de Desarrollo de Turismo Sostenible 2007-2020 (Ministerio de Turismo del Ecuador, 2007), que busca consolidar el turismo sostenible como un elemento dinamizador del desarrollo económico y social del 
Ecuador, con la participación del sector público y bajo la rectoría del Ministerio de Turismo y el involucramiento activo de otras entidades gubernamentales, como Consejos Provinciales y Municipios, hoy Gobiernos Autónomos Descentralizados (GAD); además de la participación de los actores del sector privado y comunitario a través de las Cámaras de Turismo, los gremios y las comunidades locales. Para el 2009, el boom del turismo en el Ecuador se enmarca en políticas públicas para fomentar el turismo, así como la promoción que se dio a través de la marca país Ama la Vida, y estrategias mercadológicas de gran impacto mundial, como All you need is Ecuador, Feel Again Project y Love is in the Air en conjunto con grandes inversiones privadas en hospitalidad, facilidades turísticas y la formación de talento humano especializado, lograron incrementar la actividad turística en un $19 \%$ en la llegada de turistas internacionales y en $39 \%$ el incremento del turismo interno.

Ecuador tiene una economía primaria que se sustenta principalmente en la exportación de petróleo. Sus otras exportaciones primarias no petroleras son de banano, camarón, flores, cacao y café. El componente de sus exportaciones no primarias, es decir, de bienes con algún valor añadido, apenas representa el $12 \%$ del total de sus exportaciones. Al interior de su economía, el sector más importante es el comercial el cual absorbe a casi dos tercios de la población económicamente activa. La industria representa apenas el 10\% de las actividades económicas totales del país. Al interior del país, alrededor de 60 grupos empresariales controlan el $90 \%$ de todas las actividades económicas y tienen un ingreso de aproximadamente $50 \%$ del PIB (Albuja, 2013). A pesar de que la pobreza se ha reducido de $68 \%$ en 1999 a $26 \%$ en 2012 y al $12 \%$ en 2018, el poder de los monopolios y grupos económicos se ha fortalecido, lo que ha implicado un fuerte proceso de concentración y centralización del capital en el mercado ecuatoriano. A nivel nacional existen 5488 establecimientos de alojamiento, de los cuales 40 son de lujo, 700 son de primera categoría, 1682 son de segunda categoría, 2985 son de tercera categoría, 56 son de cuarta categoría y 25 de categoría única. A nivel nacional, existen 17325 establecimientos de alimentos y bebidas. De estos, 63 son de lujo, 1424 son de primera categoría, 3939 son de segunda categoría, 8153 son de tercera categoría y 3746 son de cuarta categoría. Las ventas en el servicio turístico entre los años 2010 y 2018 fueron de 5857.9 millones de dólares. El mayor nivel de ventas se registró en el año 2015 con 1018.5 millones de dólares.

En el año 2015 había 35476 personas empleadas en las actividades de alojamiento (se registran 37518 ), mientras que las actividades de alimentos y bebidas generaron un total de 85 654 empleos. De las 121130 personas empleadas en total en todas estas actividades, 52.23\% eran hombres y $47.77 \%$ eran mujeres. En el periodo comprendido entre los años 2010 y 2018 se han generado 1905.47 millones de dólares en créditos para el sector turístico (CFN, 2018).

Con todos estos antecedentes, se decide identificar las modalidades turísticas y destinos que se ofertan en el Cantón Puerto Quito, investigación que será la base de la planificación y gestión de nuevos productos turísticos, así como de su comercialización en el ámbito local e internacional, para lo cual se plantearon cinco objetivos específicos:

- Identificar expresiones culturales potenciales para la actividad turística en el cantón Puerto Quito. 
- Cuantificar, desde la tendencia turística, las diferentes modalidades existentes en Puerto Quito.

- Identificar la participación empresarial en la operación de modalidades turísticas.

- Caracterizar el sistema turístico territorial de los espacios estudiados.

- Identificar las modalidades turísticas por ecosistemas.

\section{Aproximación teórica a las modalidades y destinos}

Una de las actividades más gratificantes que realiza el ser humano es la de viajar con un propósito de esparcimiento, de placer; si a esto le sumamos la posibilidad de experimentar nuevas emociones, de conocer paisajes diferentes y de entrar en contacto de una manera directa con otras culturas, tenemos la explicación de que se haya convertido en una necesidad de primer orden. Por otro lado, las posibilidades económicas de ciertos sectores de la población mundial han aumentado - sobre todo a partir de la segunda mitad del siglo XX - y consecuentemente ha aumentado la posibilidad de satisfacer esa necesidad. De aquí que el turismo haya sido en los últimos años uno de los sectores económicos de mayor crecimiento en el planeta.

El turismo es un sector que, además de generar trabajos, propicia la diversificación de productos agrícolas, la creación de empresas, así como el fortalecimiento cultural y patrimonial de las localidades anfitrionas.

En el mundo existen diversas formas de hacer turismo, debido a esto la tipología del turismo se sustenta en diferentes elementos para llevar a cabo una clasificación tomando como principal variable la motivación del viaje, que debido al propósito de este se podrán ordenar respectivamente dentro de las modalidades turísticas (Ibáñez y Cabrera, 2011).

La actividad turística se clasifica de acuerdo con el componente espacial, temporal o incluso con el propósito del viaje. Cada modalidad turística engloba un grupo amplio de actividades (Ibáñez y Rodríguez, 2007). En este contexto se recogen algunas de las definiciones más citadas de investigadores y académicos acerca de las modalidades turísticas hasta la actualidad.

Según Acerenza (2006), existen dos grandes modalidades: el turismo masivo y el turismo alternativo, dentro de las cuales se destacan varias tipologías necesarias para la práctica del turismo acorde a las motivaciones y preferencias del turista. El turismo masivo hace énfasis en destinos de sol y playa. Gordon (2002) menciona que, dado el extenso crecimiento del turismo en el siglo XX, fue usado el término masas, que alude a los grandes desplazamientos de personas, desplazamientos que, dicho sea de paso, generaron impactos positivos y negativos.

El turismo alternativo por su parte implica una nueva filosofía de sentir y operar el turismo; es el reflejo del cambio de tendencia en el mundo hacia lo sostenible, hacia un reencuentro saludable del hombre con la naturaleza, y un reconocimiento del valor de la interacción con la cultura rural 
(Secretaría de Turismo de México, 2004).

El Turismo Alternativo tiene su origen en 1985 cuando los organizadores de la Bolsa Internacional del Turismo de Berlín brindaron la oportunidad al movimiento ambientalista denominado Turismo Consciente y Responsable para presentar su propuesta filosófica distinta a la del turismo de masas. Se trata de una práctica turística que se caracteriza por propiciar un desarrollo del turismo en menor escala, con un mínimo impacto ambiental, una práctica turística respetuosa de los valores de las comunidades y su cultura. A este grupo se le dio la oportunidad de expresar lo que les parecía mal en el ámbito turístico, y así se produjo el primer debate en 1986. En ese momento 14 organizaciones bautizaron a esta nueva forma de hacer turismo como Turismo Consciente y Responsable, el cual finalmente sería denominado como Turismo Alternativo (Fuentes, 2019).

Lo primero que tiene que quedar muy claro en este caso es que el turismo alternativo no es en realidad una forma de turismo que responda a una motivación de los turistas. Tiene su origen en un movimiento que surge en Europa a mediados de los años ochenta, integrado por organizaciones ambientalistas, especialistas y profesionales del turismo, animados por el propósito de luchar contra las secuelas negativas del turismo en todo el mundo (Acerenza, 2010).

La modalidad turística busca ser la diferenciación que define una forma específica de hacer turismo con relación a otra, define un aspecto singular o condición de que motive la práctica del turismo y guíe a la organización de gestión de un destino o territorio a su planificación y gobernanza. Es claro que no todos los turistas practican la misma forma de visitar lugares diferentes a sus sitios de residencia, ni pernoctan en un mismo tipo de alojamiento, ni se motivan por una misma actividad, esto determina tendencias que generarán una diversidad de productos, que serán acogidas por una modalidad o modalidades en particular. Estas modalidades son el Turismo Sol y Playa, el Ecoturismo, el Turismo Rural, el Turismo Urbano y el Turismo Aventura (Fuentes, 2019).

El ecoturismo es toda "forma de turismo basado en la naturaleza en la que la motivación principal de los turistas sea la observación y apreciación de esa naturaleza o de las culturas tradicionales dominantes en las zonas naturales" (Organización Mundial del Turismo, 2010). Dentro de esta modalidad se pueden identificar algunas tendencias operadas de acuerdo a la motivación del turista, así: turismo de naturaleza, aviturismo, científico, cacería fotográfica, de lupa, entre los principales. El Turismo de Aventura, que es una de las más recientes, es definido por la Secretaría de Turismo (2004, citado en la UNID, 2014a) como aquellos viajes que se hacen con el fin de realizar actividades recreativas asociadas a desafíos impuestos por la naturaleza. En UNID (2014b) menciona que existen tres tipos de actividades que se pueden realizar dependiendo del ambiente en el que se lleva a cabo y estas son: en tierra, agua y aire (UNID, 2014).

El Turismo Rural, que es un sector emergente que surge por la necesidad de atender la demanda de los nuevos consumidores turísticos. La búsqueda de nuevas emociones (aventura, lugares históricos, contacto con la naturaleza, etc.) implica el cambio experimentado en el 
comportamiento del consumidor (Millán, López-Guzmán y Agudo, 2006). En esta modalidad por lo general se practican actividades como el turismo de base comunitaria, el agroturismo, el enoturismo, el turismo gastronómico, entre las más populares.

El Turismo Urbano toma fuerza al ser el motor de crecimiento y desarrollo de ciudades con categoría de Patrimonio Cultural de la Humanidad Mundial entregado por la Unesco y a partir de ello convertirse en un imán de visita que genera indirectamente un crecimiento económico en todas las escalas dentro y alrededor del patrimonio; ha generado impactos negativos y positivos, dependiendo el modelo de gestión local y se aprecian tendencias como el turismo naranja, turismo cultural, de dolor, histórico, de museos, religioso, entre los más populares (Fuentes, 2019).

El determinar de manera acertada las modalidades y tendencias de un territorio permite diseñar productos turísticos consolidados para su comercialización, determina cómo debe prepararse el destino para recibir turistas y excursionistas, especializa las facilidades turísticas y la infraestructura de apoyo al turista, logrando que la operación turística, ya sea formal o informal, se facilite y genere experiencias de calidad en un determinado destino.

Siendo el destino, según el Grupo de Expertos en Gestión de Destinos de la Organización Mundial del Turismo (citado en Barrado, 2004), un espacio físico en el cual un turista está al menos una noche, incluye productos turísticos tales como servicios de apoyo y atracciones y recursos turísticos que pueden ser consumidos en un recorrido de ida y vuelta el mismo día. Tiene fronteras físicas y administrativas que definen su gestión, e imágenes y percepciones que definen su posicionamiento en el mercado. El mismo que representa una base fundamental para el desarrollo del turismo (Barrado, 2004).

\section{Materiales y métodos}

El cantón Puerto Quito es uno de los ocho (8) cantones de la Provincia de Pichincha, se encuentra ubicado al noroccidente, a tres horas y 15 minutos del mismo. Según la Secretaría Nacional de Planificación y Desarrollo, divide al territorio ecuatoriano en nueve (9) agendas zonales de las cuales Pichincha, exceptuando el Distrito Metropolitano de Quito que pertenece a la Agenda Zonal 2 (SENPLADES, 2015). Este cantón limita al norte y oeste con Esmeraldas, al sur con Santo Domingo y al este con el cantón Pedro Vicente Maldonado. Políticamente el territorio contiene una sola parroquia, que se encuentra dividida en varios recintos. "El cantón tiene aproximadamente $640,70 \mathrm{Km} 2$, con una población de 20.445 cuya densidad poblacional es de 31.9 hab. $/ \mathrm{Km} 2$ lo que representa el $7.3 \%$ del territorio de la provincia de Pichincha (Instituto Nacional de Estadística y Censo, 2010).

En el cantón la agricultura representa la principal fuente de trabajo, sumando a este la ganadería que genera un comercio dinámico, ocupando el sector turismo el tercer puesto en la actividad económica, constituyéndose como una potencialidad del cantón, que aún no ha logrado tener éxito debido a la debilidad en la planificación del territorio como destino (Gobierno Autónomo descentralizado de Puerto Quito, 2015). 
Esta investigación de tipo exploratoria y descriptiva se inicia en una primera etapa basada en revisión bibliográfica de libros publicados sobre el área del conocimiento no mayor de cinco años a la fecha de búsqueda, análisis de artículos indexados en Latindex, Scielo y Scopus, además de ponencias y artículos de prensa. Se aplicaron los siguientes procedimientos: 1) ficha de observación geográfica basada en la metodología de Diagnóstico al Plan de Negocios, que contiene 12 componentes (Cabanilla, 2019), de los cuales se nombraron solo cinco para el trabajo de investigación; 2) matriz digital de jerarquización de atractivos turísticos del Ministerio de Turismo del Ecuador, en donde se calificaron por atractivo nueve componentes y 213 indicadores de acuerdo con la metodología 2018 MINTUR; 3) se aplicó la matriz de identificación de ecosistemas en base al Sistema de clasificación de ecosistemas del Ministerio del Ambiente junto con el Sistema Nacional de Información y la Secretaría Nacional de Planificación y Desarrollo, tomando en cuenta seis de los siete factores diagnóstico y enfatizando que las mismas fichas fueron aplicadas in situ con la ayuda de un Global Position System (GPS) y una aplicación móvil de medición Handy GPS; 4) se aplicó la matriz de identificación de expresiones culturales, esta ficha se basó en la guía etnográfica de Patricio Guerrero del año 2002 que estudia aspectos de la organización social y económica, idioma, representaciones artísticas, tradiciones orales, vestimenta, alimentación, patrimonios tangibles e intangibles y naturales, actividades lúdicas y su arquitectura; 5) se utilizó la matriz de selección de empresas de servicios turísticos en el territorio, la cual fue elaborada por el equipo de trabajo basándose en una matriz realizada por Quito Turismo, y 6) se aplicaron 100 encuestas a turistas una vez aplicada la fórmula finita de población conocida.

\section{Resultados y discusión}

1. Tras aplicar los seis componentes se obtuvieron los siguientes resultados: la única ruta de acceso es terrestre; tomando como referencia la capital de la provincia (Quito), existe un recorrido aproximado de 3 horas y 15 minutos con una distancia de $163 \mathrm{Km}$.

Respecto a la infraestructura de uso turístico, se pueden encontrar 15 entidades de salud, la mayor parte de la población tiene acceso a los servicios básicos y poca población tiene acceso a la educación secundaria y superior.

En términos de promoción y comercialización se encontró que existen ciertas falencias en cuanto a las páginas web ya que no se encuentran actualizadas y no se puede acceder a ellas. Finalmente, el cantón se maneja bajo la Ley de Turismo y dentro de las instituciones educativas que trabajan con el cantón están la Universidad Central del Ecuador (UCE), la Universidad de las Fuerzas Armadas (ESPE) y la Universidad Tecnológica Equinoccial (UTE), las cuales han desarrollado proyectos de tesis de grado.

2. Los seis atractivos alcanzan la jerarquía dos debido a que presentan déficits en los siguientes componentes: accesibilidad y conectividad, planta turística, higiene y seguridad turística, difusión del atractivo, recursos humanos, políticas y regulaciones, todos los cuales obtuvieron las más bajas puntuaciones. 
Tabla 1

Resultados de la ficha de jerarquización de atractivos turísticos

\begin{tabular}{lcc}
\hline Nombre del atractivo & $\begin{array}{c}\text { Resultados de la Evaluación } \\
\text { Técnica }\end{array}$ & Jerarquía \\
\hline La Cascada Azul & 46.4 & II \\
Cascada del Silencio & 33.6 & II \\
Centro recreacional y balneario & 57.9 & II \\
Río Caoní & 54.9 & II \\
Cascada de Macallares & 50.8 & II \\
Piedra de Vapor & 48.2 & II \\
Finca Integral Vill's Paradise & 48.6 & II \\
Promedio &
\end{tabular}

3. Los ecosistemas dentro de los atractivos son caracterizados por ser principalmente bosques con árboles que superan los 5 metros, con un bioclima pluviestacional, con áreas inundables en el caso de atractivos como piedra de Vapor y Centro recreacional y el balneario Río Caoní; para el resto de los atractivos expresa que son zonas no inundadas que se encuentran en el litoral del Chocó. Además, se destacan bosques siempreverdes que presentan altitudes no superiores a los 500 metros sobre el nivel del mar ( $\mathrm{msnm}$ ), sus pisos bioclimáticos son característicos de tierras bajas y pie montano.

4. Como manifestaciones culturales se obtiene que su principal representación es la marimba ya que se identifican con ella porque es sinónimo de su libertad, de manera religiosa su santo es San Antonio al cual le rinden tributo y organizan fiestas en su nombre, durante sus fiestas no solo religiosas cantan arrullos ya que significa la alegría de sus habitantes y de igual manera lo hacen en los velorios de los bebés o niños para que encuentren un mejor camino, en su gastronomía prevalece el tapao arrecho que lo hacen cuando van a realizar trabajos que demandan gran cantidad de esfuerzo físico y juegos tradicionales tales como: el cuchi-cuchi, el puente está quebrado, pata-pata, la yuca y el tun-tun. Se debe destacar la presencia de una pequeña junta libre del recinto el Cabuyal, Vía Piedra de Vapor perteneciente al grupo étnico Afroecuatoriano que migró desde la provincia de Esmeraldas.

5. Tras aplicar la ficha de registro de establecimientos se obtuvieron los siguientes resultados: Existen 30 servicios turísticos los cuales se dividen en 18 que ofrecen alimentos y bebidas y 12 servicios de alojamiento. Al realizar una comparación con el consolidado nacional del presente año se observa que existen 38 servicios turísticos de los cuales 24 ofertan el servicio de alimentos y bebidas, 13 de alojamiento y uno el servicio de casino.

Con la aplicación de la matriz de selección de empresas se identificó la existencia de dos hosterías que cuentan con una certificación de calidad, la Hostería Malacatos que cuenta con una certificación $Q$ de calidad y la Hostería La Isla con una certificación de excelencia de TripAdvisor. Existe una fuerte concentración de restaurantes y hosterías. 
Tabla 2

Servicios que oferta el cantón

\begin{tabular}{lc}
\hline Servicios que se ofertan & Cantidad \\
\hline Alimentación & 10 \\
Bar & 5 \\
Hospedaje & 4 \\
Hospedaje, alimentación, recreación & 3 \\
Hospedaje, alimentación, recreación, camping & 2 \\
Hospedaje, alimentación & 1 \\
Cafetería & 1 \\
Bar, cafetería & 1 \\
Hospedaje, Kayak & 1 \\
Camping, alimentación, recreación & 1 \\
Heladería & 1 \\
Total general & 30 \\
\hline
\end{tabular}

Del $100 \%$ de las empresas de servicios turísticos registrados, el $17 \%$ cuenta con una página web oficial o se encuentran registradas en otro tipo de redes sociales; el $20 \%$ de las empresas oferta sus servicios a través de páginas oficiales en la red social Facebook; el $63 \%$ de las empresas turísticas no oferta sus servicios por medio de páginas web.

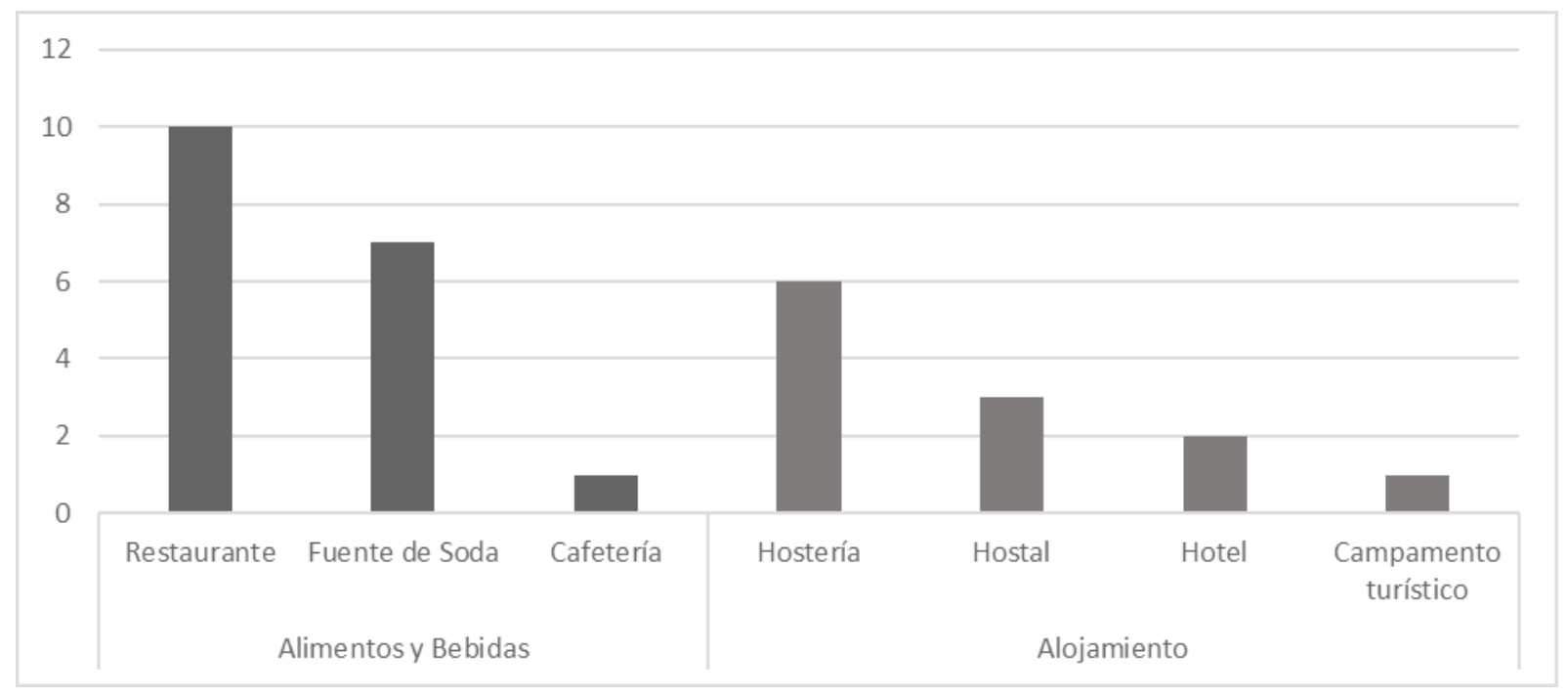

Figura 1. Resultado de las actividades.

6. Tras aplicar las encuestas a turistas del cantón Puerto Quito se determinó que predominan turistas nacionales mujeres $(67 \%)$ y hombres (33\%) con una edad de 18 a 30 años, que en 
un $66 \%$ son personas solteras, un $25 \%$ casados, el $6 \%$ en unión libre y el restante $3 \%$ son personas divorciadas, con ingresos económicos inferiores a los 500 dólares americanos. Las principales modalidades que se practican en el mencionado cantón son el turismo de Aventura y Rural (Figura 2).

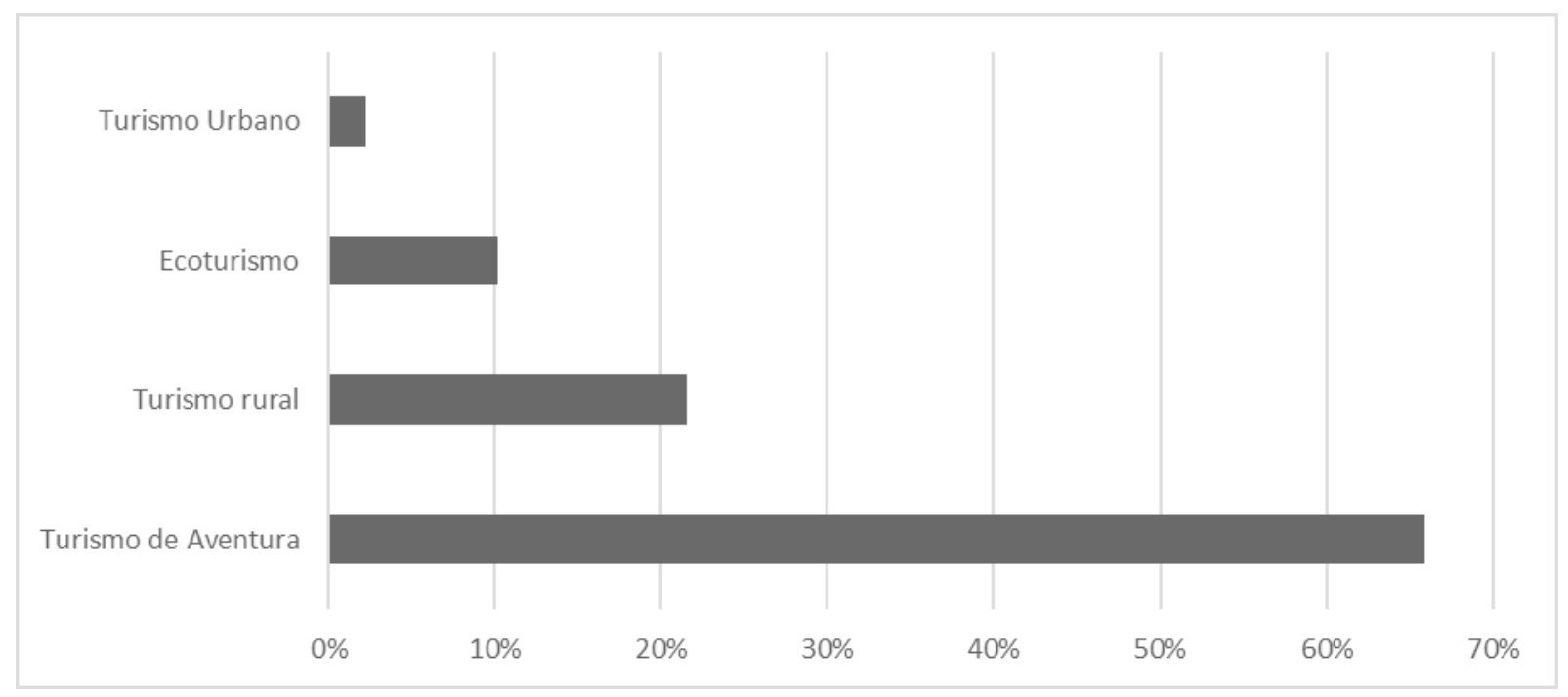

Figura 2. Tipos de modalidades.

De las modalidades elegidas, las principales tendencias turísticas identificadas son: turismo deportivo ( $77 \%$ ) con actividades como las de visita a ríos y cascadas, visita a balnearios, senderismo, caminata y excursiones; el agroturismo (13\%) como segunda tendencia predominante y actividades como visita a haciendas y fincas y la tendencia del aviturismo (11\%) con actividades como observación de flora y fauna (Figura 3).

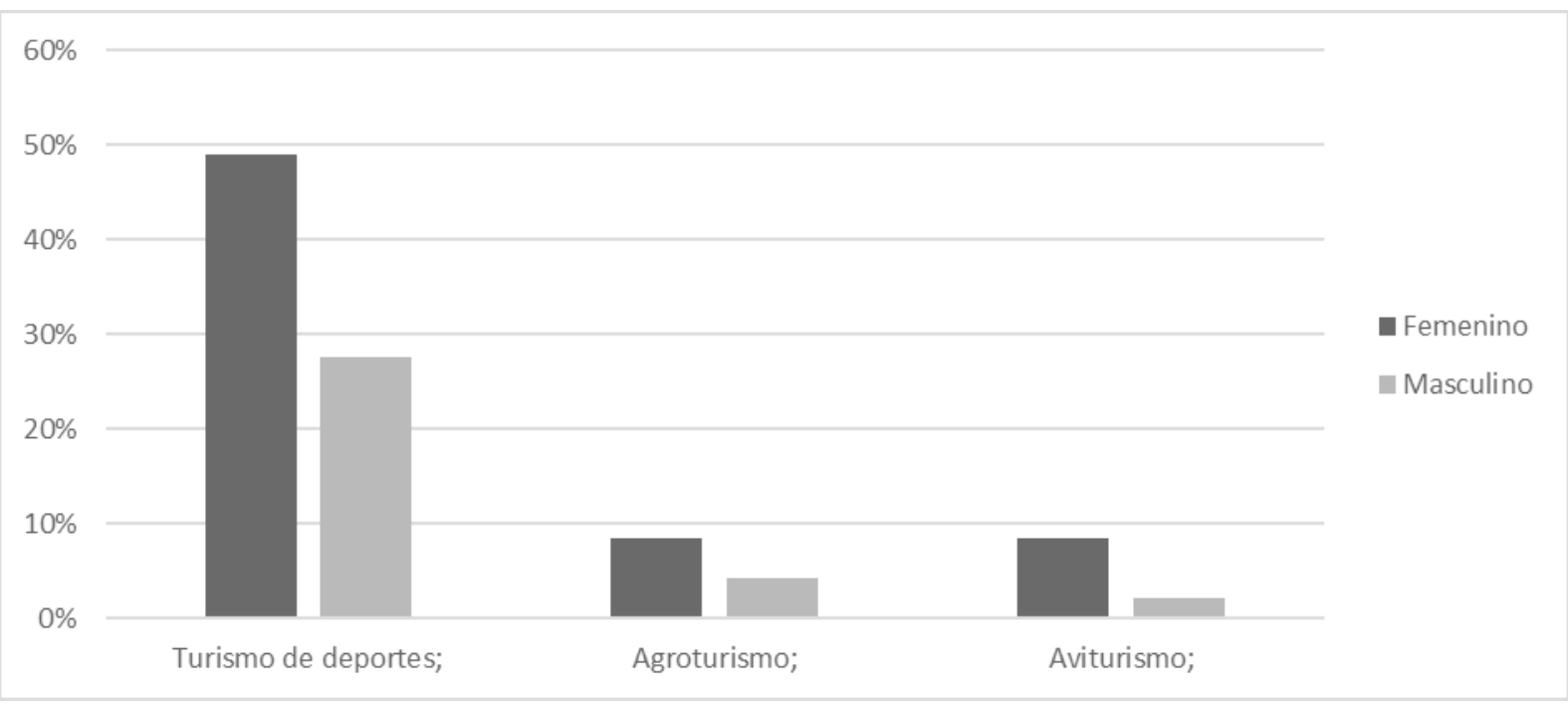

Figura 3. Tipos de tendencias. 
Por otro lado, en la Figura 4 se puede evidenciar que según lo encuestado los turistas nacionales tienen un presupuesto de gasto como mínimo de 10 y un máximo de 700 dólares americanos mismo que refleja un promedio de 152.84 dólares americanos para servicios turísticos.

Los visitantes del cantón Puerto Quito permanecen en el territorio en promedio de 1 a 3 días con un $94 \%$ y un porcentaje mínimo del $4 \%$ pernocta de 4 a 7 días, acompañados en su mayoría de familiares y amigos correspondientes al $45 \%$ y $42 \%$ respectivamente y el $13 \%$ va junto con su pareja.

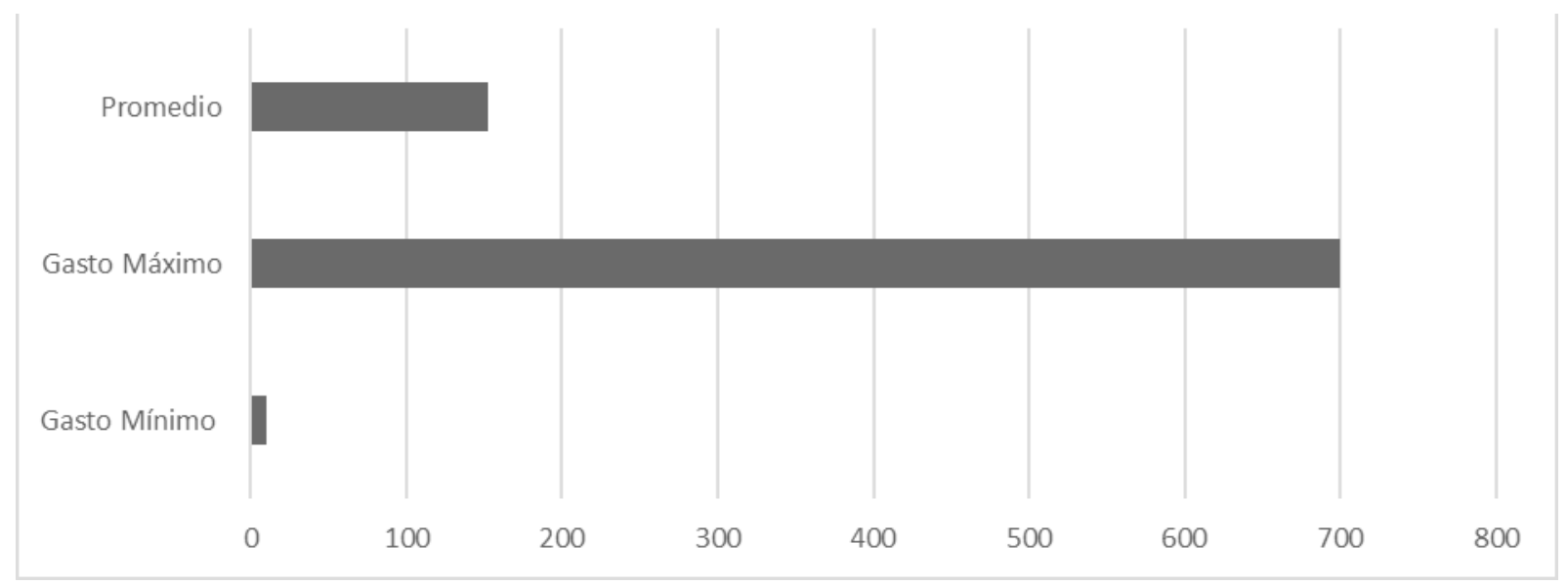

Figura 4. Gasto en turismo.

Sus principales medios de transporte son: el $49 \%$ transporte privado, el $40 \%$ público, el $9 \%$ transporte turístico y tan solo el $2 \%$ que responde a la suma de transporte como la moto o a pie usados para llegar al destino, del que obtuvieron información por recomendaciones de familiares y amigos con el $61 \%$, por redes sociales en un $25 \%$, seguidos de un $13 \%$ a través del internet y tan solo el $1 \%$ por medio de la televisión como se muestra en la Figura 5.

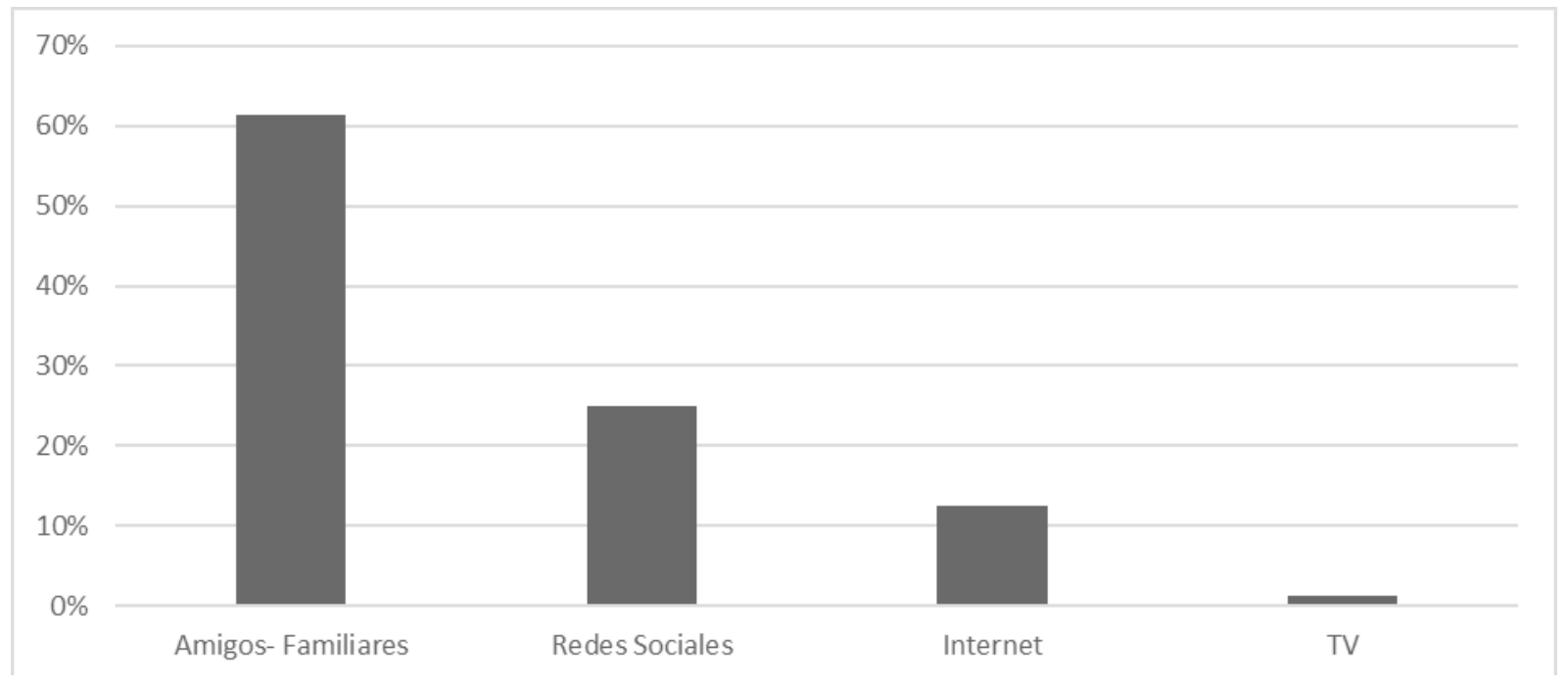

Figura 5. Medios de conocimiento de destino. 
El $51 \%$ de los turistas dicen no reconocer una ruta turística que les permita llegar al cantón contra un $49 \%$ que asegura reconocerla (Figura 6 ).

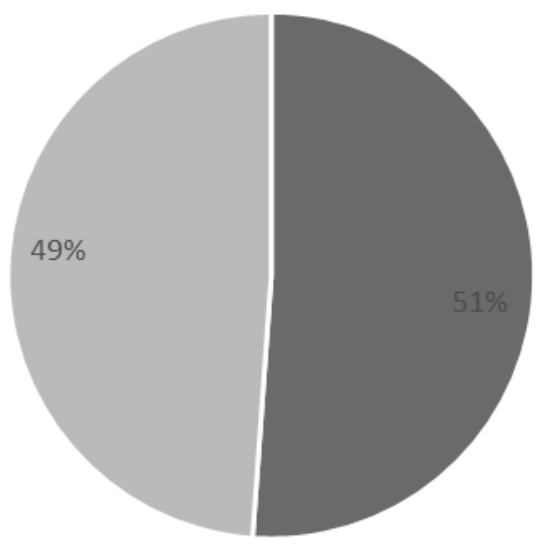

- No

- Sí

Figura 6. Reconocimiento de Ruta Turística.

\section{Conclusiones}

La planificación turística del cantón es empírica, no se pudo evidenciar una directriz que direccione la planificación turística del territorio.

La comercialización y promoción turística es débil, no se encuentra información relevante, actualizada y detallada de la oferta turística.

El cantón Puerto Quito oferta las modalidades de turismo de aventura y turismo rural, con una fuerte tendencia a realizar actividades como deportes extremos, senderismo, visita a balnearios y fincas.

Por la ubicación geográfica de Puerto Quito, la zona del Litoral Choco, todos sus atractivos turísticos poseen las mismas características en cuanto a los ecosistemas.

El patrimonio turístico natural y cultural en el cantón es relevante y se muestra como potencial impulsor del crecimiento y desarrollo económico del territorio teniendo como eje al turismo.

En el cantón Puerto Quito no prestan servicios operadoras turísticas, y los servicios turísticos de alojamiento y restauración se encuentran ubicados únicamente en el trayecto de la avenida principal, lo que frena y limita el acceso a algunos lugares pintorescos del territorio. 


\section{Referencias bibliográficas}

Acerenza, M. A. (2006). Conceptualización, origen y evolución del turismo. Recuperado de https://www.entornoturistico.com/wp-content/uploads/2017/11/Conceptualizaci\%C3\%B3n-origen-y-evoluci\%C3\%B3n-del-turismo-de-Miguel-Acerenza-PDF.pdf

Barrado, D. A. (2004). El concepto de destino turístico. Una aproximación geográfico-territorial. Estudios Turísticos, (160), 45-68. Recuperado de http://www.iet.tourspain.es/img-iet/Revistas/RET-160-2004pag45-68-91963.pdf

Cabanilla, E. (2019). Metodología de diagnóstico al plan de negocios. Quito, Ecuador: Universidad Central del Ecuador.

Comisión Económica para América Latina y el Caribe. (2014). Análisis de la cadena del turismo en el Ecuador. Quito, Ecuador.

Fuentes, A. (2019). Turismo Alternativo. Quito, Ecuador.

Gobierno Autónomo descentralizado de Puerto Quito. (2015). Plan de ordenamiento territorial. Quito: Gobierno Autónomo descentralizado de Puerto Quito.

Gordon, B. M. (2002). El turismo de masas: un concepto problemático en la historia del siglo XX. Historia Contemporánea, (25), 125-156. Recuperado de http://www.ehu.eus/ojs/index.php/HC/article/view/5928

Ibáñez, R. y Cabrera, C. (2011). Teoría General del Turismo: un enfoque global y nacional. Recuperado de http:// uabcs.mx/difusion2017/files/libros/pdf/184_20160908023838.pdf?fbclid=lwAR3GVw87eDvOPfkTh--DNRnUUNrk9x4rRICpLI5yDRyQycNUPpd2RyqY-O8

Instituto Nacional de estadística y censo. (2010). Resultados del Censo de 2010 de población y vivienda en el Ecuador. Quito.

Millán, M. G., López-Guzmán, T. y Agudo, E. (2006). El turismo rural como agente económico: desarrollo y distribución de la renta en la zona de Priego de Córdoba. CIRIEC-España, Revista de Economía Pública, Social y Cooperativa, (55), 167-192. Recuperado de http://www.redalyc.org/articulo.oa?id=17405507

Ministerio de Turismo. (2004). Estrategia Promocional Cuatro Mundos. Quito.

Ministerio de Turismo. (2007). Plan Estratégico de Desarrollo de Turismo Sostenible para Ecuador. "PLANDETUR 2020". Recuperado de https://jorgepaguay.files.wordpress.com/2012/03/plandetur.pdf

Organización Mundial del Turismo. (2010). Organización Mundial del Turismo. Recuperado de http://www2. unwto.org/es/content/ecoturismo-y-areas-protegidas 
Peña, E. (2015). El turismo urbano como agente de transformación del "producto ciudad" (tesis de maestría). Universidad Politécnica de Valencia, Valencia, España.

Prefectura de Pichincha. (29 de agosto de 2017). Prefectura de Pichincha. Recuperado de Puerto Quito: https:// www.pichincha.gob.ec/cantones/puerto-quito

SENPLADES. (2015). Agenda Zonal Zona 4-Pacífico. Ecuador: Senplades.

UNID (2014). Turismo de Aventura. México: Universidad Interamericana para el Desarrollo. Recuperado de https://moodle2.unid.edu.mx/dts_cursos_mdl/lic/AET/TA/S01/TA01_Lectura.pdf 
FUENTES MORENO · RIVAS RAMÍREZ • ARCENTALES AULESTIA · SALAZAR AGUILAR 